\title{
Dynamic Effective Properties of Particle-Reinforced Composites with Viscoelastic Matrix
}

\author{
P.J. WEI ${ }^{1,2}$ \\ ${ }^{1}$ LNM (State Key Laboratory of Nonlinear Mechanics), Institute of Mechanics, Chinese Academy of \\ Sciences, Beijing, P.R. China \\ ${ }^{2}$ School of Applied Sciences, University of Sciences and Technology Beijing, Beijing, P.R. China; \\ E-mail:weipj@126.com
}

(Received 11 February 2004; accepted in revised form 11 February 2004)

\begin{abstract}
The frequency-dependent dynamic effective properties of the particle-reinforced composites with the viscoelastic matrix are studied. Several equations to predict the effective wavenumber of the coherent plane waves propagating through particle-reinforced composites are discussed and the equation given by Gubernatis, J.E., ['Effects of microstructure on speed and attenuation of elastic waves in porous materials', Wave Motion, 6, 1984, 579-589] based on the independent scattering approximation is used in this paper. The effective phase velocity, the effective attenuation and the effective elastic moduli are evaluated. Numerical calculations are carried out for two kinds of composites, namely, Lead-Epoxy and Glass-Epoxy and the numerical results show that the frequency-dependent dynamic effective properties are related to both the multiple scattering effects among the distributed particles and the viscous dissipative effects of the viscoelastic matrix. However, these effects in the composites with distributed heavy particles (lead) and light particles (glass) are of evidently different features.
\end{abstract}

Key words: dynamic effective properties, multiple scattering, coherent plane waves, configurational average, viscoelastic matrix

\section{Introduction}

The determination of the effective propagation constants of waves propagating through composite materials has been a subject which attracted a considerable attention (Foldy, 1945; Lax, 1951; Varadan, 1985; Datta, 1988; Shindo, 1995; Kanaun, 2000). Foldy studied early the effective wavenumber of a scalar wave propagating through an inhomogeneous medium with distributed particles based on the multiple scattering theory. In this theory, a set of equations in hierarchy, each containing more statistical distribution information than those preceding, is involved. In order to truncate these equations to obtain an approximate solution, a self-consistent approximation is given by Foldy. Later, in order to consider better the distribution correlation between two particles the well-known "quasi-crystalline approximation" is proposed by Lax. Varadan et al. (1985) extended the multiple scattering theory of a scalar wave to the elastic waves. Further, for the elastic longitudinal and shear waves propagating through the composite medium, Gubernatis 
(1984) gave the coherent plane wave equations based on the independent scattering approximation. It is evident that all the approximations mentioned above are aimed at simplifying the interaction among the particles. On the other hand, the interaction among the distributed particles can be described approximately by assuming that each particle is embedded in the effective medium. It is usually called the effective medium approache and is employed by Berryman (1980), Sabina and Willis (1988), Kanaun (2000) and others. The multiple scattering theory and the effective medium approach are based on different assumptions to simplify calculations, and thus, generally speaking, will give different results when applied to a given composite medium. Nevertheless, same qualitative conclusions are obtained, namely, the coherent plane waves are of attenuation nature although the matrix and the distributed particles are both elastic materials. If the viscoelasticity of matrix is considered, then the material dissipative effects and the multiple scattering effects are both contributed to the attenuation of waves. However, most of investigations up to now are given to elastic matrix and particles. In the present work, the material dissipation is taken into account. The different effects of the material dissipation and the multiple scattering on waves propagating through composites are our main concerns. The outline of the paper is as follows: in Section 2, the scattering problem of an elastic sphere embedded in a viscoelastic matrix is studied and the far-field scattering amplitudes are formulated. In Section 3, several equations to predict the effective wavenumbers of the coherent plane waves by using the forward scattering amplitudes of an individual particle are discussed. In Section 4, the effective propagation constants and the effective elastic moduli are studied numerically for Lead-Epoxy and Glass-Epoxy composites. The material dissipation effects and the multiple scattering effects in the composites with distributed heavy and light particles are compared. Finally, conclusions are given in Section 5.

\section{The Scattered Waves by an Elastic Sphere Embedded in a Viscoelastic Matrix}

Consider a spherical inclusion of radius $a$ embedded in a viscoelastic matrix. The lam'e constants and the mass densities of the inclusion are denoted by $\left(\lambda_{1}, \mu_{1}, \rho_{1}\right)$. Due to the viscoelasticity of matrix, the $\operatorname{lam}^{\prime} e$ constants of matrix are complexvalued and frequency-dependent in the time harmonic case. The complex-valued and frequency-dependent $l a m^{\prime} e$ constants and the mass densities of the viscoelastic matrix are denoted by $\left(\hat{\lambda}_{2}(\omega), \hat{\mu}_{2}(\omega), \rho_{2}\right)$. The geometry is depicted in Figure 1 , where $(x, y, z)$ is the right-handed Cartesian coordinate system with the origin at the center of spherical inclusion and $(r, \theta, \phi)$ is the corresponding spherical polar coordinate. The incident plane longitudinal and shear waves ( $\mathrm{P}$ and $\mathrm{S}$ waves) of circular frequency $\omega$ are assumed to propagate along $z$-axis and can be expressed as the displacement vector

$$
\mathbf{u}^{\mathrm{i}}=\mathbf{a} e^{\mathrm{i}\left(k_{\mathrm{p}}^{0} z-\omega t\right)}+\mathbf{b} e^{\mathrm{i}\left(k_{\mathrm{s}}^{0} z-\omega t\right)}
$$



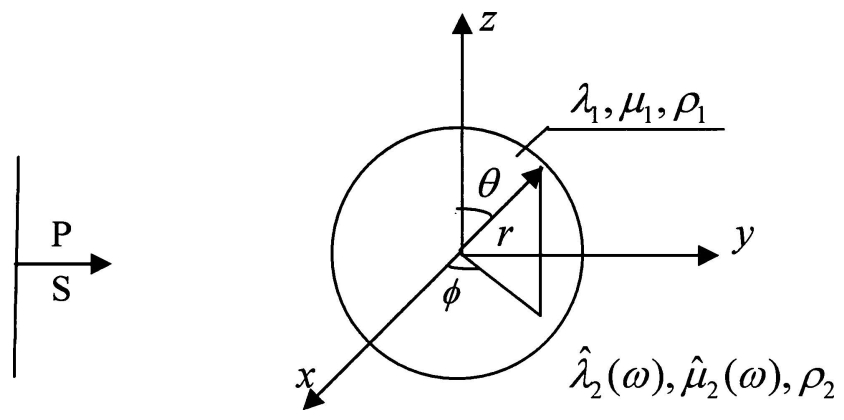

Figure 1. The scattered waves by a spherical inclusion embedded in a viscoelastic matrix.

where $\mathbf{a}=a \mathbf{e}_{z}$ and $\mathbf{b}=b \mathbf{e}_{x}$ are the polarization vectors of the incident $\mathrm{P}$ and $\mathrm{S}$ waves, respectively. $\mathbf{e}_{z}$ and $\mathbf{e}_{x}$ are the unit coordinate vectors. $k_{\mathrm{p}}^{0}$ and $k_{\mathrm{s}}^{0}$ are the complex-valued wave numbers of the incident $\mathrm{P}$ and $\mathrm{S}$ waves, respectively. These complex-valued wave numbers result from the intrinsic dissipation of a viscoelastic matrix with their imaginary parts related to the attenuation of waves. When the incident waves impinge the elastic inclusion, the scattered waves in the matrix and the transmitted waves in the inclusion are induced.

In order to evaluate the scattered wave fields in the matrix, it is necessary to take into account the transmitted waves in the inclusion and the continuous conditions of displacements and tractions across the interface. It is noticed that the wavenumbers of the waves in the elastic inclusion are real-valued. But the wavenumbers of the waves propagating through the matrix are complex-valued due to the viscosity of matrix. The constitutive equations of a viscoelastic material can be expressed in the Stieltjes integral form

$$
\begin{aligned}
s_{i j}(t) & =\int_{-\infty}^{t} 2 G(\tau) \dot{e}_{i j}(t-\tau) d \tau, \\
\sigma_{k k}(t) & =\int_{-\infty}^{t} 3 K(\tau) \dot{\varepsilon}_{k k}(t-\tau) d \tau .
\end{aligned}
$$

It follows by Fourier integral transformation

$$
\begin{aligned}
\bar{s}_{i j}(\omega) & =2 \hat{G}(\omega) \bar{e}_{i j}(\omega), \\
\bar{\sigma}_{k k}(\omega) & =3 \hat{K}(\omega) \bar{\varepsilon}_{k k}(\omega),
\end{aligned}
$$

where $\varepsilon_{i j}, \sigma_{i j}, e_{i j}$ and $s_{i j}$ are the strain, the stress, the deviatoric strain and the deviatoric stress, respectively. And $\bar{\varepsilon}_{i j}, \bar{\sigma}_{i j}, \bar{e}_{i j}$ and $\bar{s}_{i j}$ are their corresponding Fourier transformation, i.e. $\bar{f}(\omega)=F(f(t))$. The shear and bulk complex moduli are related to the shear and bulk relaxation functions, $G(t)$ and $K(t)$, by

$$
\begin{aligned}
& \hat{G}(\omega)=\mathrm{i} \omega F(G(t)), \\
& \hat{K}(\omega)=\mathrm{i} \omega F(K(t)) .
\end{aligned}
$$


Then, the lam'e constants of a viscoelastic material are obtained by $\hat{\lambda}(\omega)=\hat{K}(\omega)-2 / 3 \hat{G}(\omega)$ and $\hat{\mu}(\omega)=\hat{G}(\omega)$. The equations of wave motion in a homogeneous elastic or viscoelastic medium are expressed as

$$
k_{\mathrm{p}}^{-2} \nabla \nabla \cdot \mathbf{u}-k_{\mathrm{s}}^{-2} \nabla \times \nabla \times \mathbf{u}+\mathbf{u}=0,
$$

where $\mathbf{u}(x, y, z, t)$ is the time harmonic displacement vector. For convenience, the time harmonic factor $e^{-\mathrm{i} \omega t}$ is omitted in the following discussion but understood. $k_{\mathrm{p}}=\omega / \sqrt{(\lambda+2 \mu) / \rho}$ and $k_{\mathrm{s}}=\omega / \sqrt{\mu / \rho}$ (real-valued for an elastic medium and complex-valued for a viscoelastic medium) are the wavenumbers of the longitudinal and the shear waves, respectively. $\nabla$ is the gradient operator. It is known that the general form of the solution of Equation (5) can be expressed as

$$
\mathbf{u}=\nabla \Phi+\nabla \times(\Psi r) \mathbf{e}_{\mathbf{r}}+\nabla \times \nabla \times(\Pi r) \mathbf{e}_{\mathbf{r}},
$$

where the scalar potentials $\Phi, \Psi$ and $\Pi$ are the solutions of the scalar Helmholtz equations

$$
\left(\nabla^{2}+k^{2}\right)(\Phi, \Psi, \Pi)=0,
$$

(where $\nabla^{2}$ is the Laplacian operator) and can be expressed in the series form

$$
(\Phi, \Psi, \Pi)=\sum_{n=0}^{\infty} \sum_{m=0}^{ \pm n} C_{n m} Z_{n}^{q}(k r) P_{n}^{m}(\cos \theta) e^{\mathrm{i} m \phi} \quad(q=1 \text { or } 3),
$$

where $C_{n m}$ is the expansion coefficient. $P_{n}^{m}(\cos \theta)$ is the associated Legendre function and the symbol $Z_{n}^{q}(k r)$ stands for the spherical Bessel function $j_{n}(k r)$ for $q=1$ and the spherical Hankel function $h_{n}^{(1)}(k r)$ for $q=3$.

In order to meet the radial conditions at infinity and to keep finite value of displacement at the center of inclusion, the potentials of the scattered waves and the transmitted waves can be expressed as

$$
\begin{aligned}
\Phi^{\mathrm{s}} & =\sum_{n=0}^{\infty} \sum_{m=0}^{ \pm n} A_{m n}^{\mathrm{s}} h_{n}^{(1)}\left(k_{\mathrm{p}} r\right) P_{n}^{m}(\cos \theta) e^{\mathrm{i} m \phi} \\
\Psi^{\mathrm{s}} & =\sum_{n=0}^{\infty} \sum_{m=0}^{ \pm n} B_{m n}^{\mathrm{s}} h_{n}^{(1)}\left(k_{\mathrm{s}} r\right) P_{n}^{m}(\cos \theta) e^{\mathrm{i} m \phi} \\
\Pi^{\mathrm{s}} & =\sum_{n=0}^{\infty} \sum_{m=0}^{ \pm n} C_{m n}^{\mathrm{s}} h_{n}^{(1)}\left(k_{\mathrm{s}} r\right) P_{n}^{m}(\cos \theta) e^{\mathrm{i} m \phi} \\
\Phi^{\mathrm{t}} & =\sum_{n=0}^{\infty} \sum_{m=0}^{ \pm n} A_{m n}^{\mathrm{t}} j_{n}\left(k_{\mathrm{p}} r\right) P_{n}^{m}(\cos \theta) e^{\mathrm{i} m \phi}
\end{aligned}
$$




$$
\begin{aligned}
\Psi^{\mathrm{t}} & =\sum_{n=0}^{\infty} \sum_{m=0}^{ \pm n} B_{m n}^{\mathrm{t}} j_{n}\left(k_{\mathrm{s}} r\right) P_{n}^{m}(\cos \theta) e^{\mathrm{i} m \phi}, \\
\Pi^{\mathrm{t}} & =\sum_{n=0}^{\infty} \sum_{m=0}^{ \pm n} C_{m n}^{\mathrm{t}} j_{n}\left(k_{\mathrm{s}} r\right) P_{n}^{m}(\cos \theta) e^{\mathrm{i} m \phi},
\end{aligned}
$$

where $A_{m n}^{\alpha}, B_{m n}^{\alpha}, C_{m n}^{\alpha}(\alpha=\mathrm{s}, \mathrm{t})$ are the unknown expansion coefficients. Similarly, we can expand the incident waves

$$
\begin{aligned}
\Phi^{\mathrm{i}} & =\frac{a}{\mathrm{i} k_{\mathrm{p}}^{0}} e^{\mathrm{i} k_{\mathrm{p} 0} z}=\frac{a}{\mathrm{i} k_{\mathrm{p}}^{0}} \sum_{n=0}^{\infty} \sum_{m=0}^{ \pm n}(2 n+1) \mathrm{i}^{n} \delta_{m 0} j_{n}\left(k_{\mathrm{p}}^{0} r\right) P_{n}^{m}(\cos \theta) e^{\mathrm{i} m \phi} \\
& =\sum_{n=0}^{\infty} \sum_{m=0}^{ \pm n} A_{m n}^{\mathrm{i}} j_{n}\left(k_{\mathrm{p}}^{0} r\right) P_{m}^{n}(\cos \theta) e^{\mathrm{i} m \phi} \\
\Psi^{\mathrm{i}} & =\frac{b}{\mathrm{i} k_{\mathrm{s}}^{0}} \sum_{n=0}^{\infty} \sum_{m=0}^{ \pm n} \frac{2 n+1}{2 n(n+1)}\left(\delta_{m, 1}+n(n+1) \delta_{m,-1}\right) \mathrm{i}^{n-1} j_{n}\left(k_{\mathrm{s}}^{0} r\right) P_{n}^{m}(\cos \theta) e^{\mathrm{i} m \phi} \\
& =\sum_{n=0}^{\infty} \sum_{m=0}^{ \pm n} B_{m n}^{\mathrm{i}} j_{n}\left(k_{\mathrm{s}}^{0} r\right) P_{m}^{n}(\cos \theta) e^{\mathrm{i} m \phi}, \\
\Pi^{\mathrm{i}} & =\frac{b}{\left(\mathrm{i} k_{\mathrm{s}}^{0}\right)^{2}} \sum_{n=0}^{\infty} \sum_{m=0}^{ \pm n} \frac{2 n+1}{2 n(n+1)}\left(\delta_{m, 1}-n(n+1) \delta_{m,-1}\right) \mathrm{i}^{n-1} j_{n}\left(k_{\mathrm{s}}^{0} r\right) P_{n}^{m}(\cos \theta) e^{\mathrm{i} m \phi} \\
& =\sum_{n=0}^{\infty} \sum_{m=0}^{ \pm n} C_{m n}^{\mathrm{i}} j_{n}\left(k_{\mathrm{s}}^{0} r\right) P_{m}^{n}(\cos \theta) e^{\mathrm{i} m \phi} .
\end{aligned}
$$

the unknown expansion coefficients $\left(A_{m n}^{\alpha}, B_{m n}^{\alpha}, C_{m n}^{\alpha}\right)(\alpha=s, t)$ can be related to the known expansion coefficients $\left(A_{n}^{\mathrm{i}}, B_{n}^{\mathrm{i}}, C_{n}^{\mathrm{i}}\right)$ by the boundary conditions. The boundary conditions that the displacements and the tractions are continuous across the interface at $r=a$ can be expressed as

$$
u_{\beta}^{\mathrm{i}}(a)+u_{\beta}^{\mathrm{s}}(a)=u_{\beta}^{\mathrm{t}}(a), \quad \sigma_{r \beta}^{\mathrm{i}}(a)+\sigma_{r \beta}^{\mathrm{s}}(a)=\sigma_{r \beta}^{\mathrm{t}}(a) \quad(\beta=r, \theta, \phi) .
$$

Then, we can express the unknown expansion coefficients by matrix form

$$
\left(A_{n}^{\mathrm{s}}, B_{n}^{\mathrm{s}}, C_{n}^{\mathrm{s}}, A_{n}^{\mathrm{t}}, B_{n}^{\mathrm{t}}, C_{n}^{\mathrm{t}}\right)=\mathbf{T} \cdot\left(A_{n}^{\mathrm{i}}, B_{n}^{\mathrm{i}}, C_{n}^{\mathrm{i}}\right),
$$

where $\mathbf{T}$ is so-called $T$-matrix which is dependent on the properties of matrix and inclusion. Further, from the asymptotic expression of radial function,

$$
h_{n}(k r) \sim \frac{1}{k r} e^{\mathrm{i}\left[k r-\frac{1}{2}(n+1) \pi\right]}+o\left(\frac{1}{r}\right) \quad(r \rightarrow \infty),
$$


we can obtain the displacement of the scattered waves in the far-field asymptotically

$$
\begin{aligned}
u_{r}^{\mathrm{s}} \sim & \frac{1}{r} e^{\mathrm{i} k_{\mathrm{p}}^{0} r} \sum_{n=0}^{\infty} \sum_{m=0}^{ \pm n} \mathrm{i} A_{m n}^{\mathrm{s}} e^{-\mathrm{i} \frac{1}{2}(n+1) \pi} P_{n}^{m}(\cos \theta) e^{\mathrm{i} m \phi}+o\left(\frac{1}{r}\right) \\
= & \frac{F_{r}(\theta, \phi)}{r} e^{\mathrm{i} k_{\mathrm{p}}^{0} r}+o\left(\frac{1}{r}\right) \\
u_{\theta}^{\mathrm{s}} \sim & \frac{1}{r} e^{\mathrm{i} k_{\mathrm{s}}^{0} r} \sum_{n=0}^{\infty} \sum_{m=0}^{ \pm n} \mathrm{i} e^{-\mathrm{i} \frac{1}{2}(n+1) \pi}\left[B_{m n}^{\mathrm{s}} \frac{m}{k_{\mathrm{s}}^{0} \sin \theta} P_{n}^{m}(\cos \theta)\right. \\
& \left.+C_{m n}^{\mathrm{s}} \frac{d}{d \theta} P_{n}^{m}(\cos \theta)\right] e^{\mathrm{i} m \phi}+o\left(\frac{1}{r}\right) \\
= & \frac{F_{\theta}(\theta, \phi)}{r} e^{\mathrm{i} k_{\mathrm{s}}^{0} r}+o\left(\frac{1}{r}\right), \\
u_{\phi}^{\mathrm{s}} \sim- & \frac{1}{r} e^{\mathrm{i} k_{\mathrm{s}}^{0} r} \sum_{n=0}^{\infty} \sum_{m=0}^{ \pm n}\left[\frac{B_{m n}^{\mathrm{s}}}{k_{\mathrm{s}}^{0}} \frac{d}{d \theta} P_{n}^{m}(\cos \theta)\right. \\
& \left.+\frac{m}{\sin \theta} C_{m n}^{\mathrm{s}} P_{n}^{m}(\cos \theta)\right] e^{-\mathrm{i} \frac{1}{2}(n+1) \pi} e^{\mathrm{i} m \phi}+o\left(\frac{1}{r}\right) \\
= & \frac{F_{\phi}(\theta, \phi)}{r} e^{\mathrm{i} k_{\mathrm{s}}^{0} r}+o\left(\frac{1}{r}\right),
\end{aligned}
$$

where $F_{r}(\theta, \phi), F_{\theta}(\theta, \phi)$ and $F_{\phi}(\theta, \phi)$ are called the far-field scattered amplitudes. It is noticed that the far-field scattered amplitudes are azimuth-dependent. The far-field scattered amplitudes at azimuthal angle, $\theta=0$, are called the forward scattering amplitudes, namely,

$$
\begin{aligned}
& F_{r}(0, \phi)=\sum_{n=0}^{\infty}(-\mathrm{i})^{n} A_{0 n}^{\mathrm{s}} \\
& F_{\theta}(0, \phi)=\sum_{n=0}^{\infty} \frac{(-\mathrm{i})^{n}}{2}\left[\frac{n(n+1)}{k_{\mathrm{s} 0}} B_{1 n}^{\mathrm{s}} e^{\mathrm{i} \phi}+\frac{1}{k_{\mathrm{s} 0}} B_{-1 n}^{\mathrm{s}} e^{-\mathrm{i} \phi}\right] \\
& F_{\phi}(0, \phi)=\sum_{n=0}^{\infty} \frac{\mathrm{i}(-\mathrm{i})^{n}}{2}\left[n(n+1) C_{1 n}^{\mathrm{s}} e^{\mathrm{i} \phi}+C_{-1 n}^{\mathrm{s}} e^{-\mathrm{i} \phi}\right]
\end{aligned}
$$

\section{Dynamic Effective Properties of the Particle-Reinforced Composites}

We now consider the composite materials of volume $V$ in which $N$ inclusions are randomly distributed. If the positions of these inclusions, denoted by the random variables $\left(\mathbf{r}_{1}, \mathbf{r}_{2}, \ldots, \mathbf{r}_{N}\right)$, are given we shall say that we have a particular configuration of the scatterers. The joint probabilities distribution, denoted by $p\left(\mathbf{r}_{1}, \mathbf{r}_{2}, \ldots, \mathbf{r}_{N}\right)$, represents the probability of finding the scatterers in this configuration. In light 
of the chain rule of the conditional probabilities, the distribution function can be written as

$$
\begin{aligned}
p\left(\mathbf{r}_{1}, \mathbf{r}_{2} \ldots \mathbf{r}_{N}\right) & =p\left(\mathbf{r}_{i}\right) p\left(\mathbf{r}_{1}, \mathbf{r}_{2}, \ldots, \mathbf{r}_{i-1}, \mathbf{r}_{i+1}, \ldots, \mathbf{r}_{N} \mid \mathbf{r}_{i}\right) \\
& =p\left(\mathbf{r}_{i}\right) p\left(\mathbf{r}_{1}, \mathbf{r}_{2}, \ldots \ldots, \mathbf{r}_{N} \mid \mathbf{r}_{i}\right),
\end{aligned}
$$

where the vertical lines in the arguments stands for the conditional probability distribution with the scatterer positioned at $\mathbf{r}_{i}$ hold fixed. Symbol "'"means the absence of one inclusion. Due to the indistinguishability of inclusions, the distribution function $p\left(\mathbf{r}_{1}, \mathbf{r}_{2}, \ldots, \mathbf{r}_{N}\right)$ is symmetric in its arguments. If the composite medium is statistically uniform, then, the positions of each inclusion are equally probable within the volume $V$, namely, its distribution is uniform with density

$$
p\left(\mathbf{r}_{i}\right)=\frac{1}{V} \quad(i=1,2,3, \ldots, N) .
$$

If $j$-th inclusion is held fixed, the distribution of any one inclusions around $j$-th inclusion will be spherical symmetric, namely, $p\left(\mathbf{r}_{i} \mid \mathbf{r}_{j}\right)$ is a function of $r_{i j}=\left|\mathbf{r}_{i}-\mathbf{r}_{j}\right|$ alone, and can be generally written as

$$
p\left(\mathbf{r}_{i} \mid \mathbf{r}_{j}\right)=\frac{1}{V}\left[1-g\left(r_{i j}\right)\right]
$$

where $g\left(r_{i j}\right)$ is called the pair correlation function. The pair correlation function for an ensemble of particles depends on the nature and the range of interparticle forces. To obtain an expression for the pair correlation function, one needs a description of the interparticle force. In general, it is assumed that the scatterers behave like effective "hard" sphere. Due to the impossibility of interpenetration of inclusions and their independence when they are infinitely apart, two restrictions are obtained

$$
g\left(r_{i j}\right)= \begin{cases}1, & r_{i j} \leq 2 a \\ 0, & r_{i j} \rightarrow \infty\end{cases}
$$

The determination of the pair correlation function is usually involved and resorts to the statistic mechanics (Varadan, 1985). The configurational average of a random function $f\left(\mathbf{r} ; \mathbf{r}_{1}, \ldots, \mathbf{r}_{N}\right)$ is defined by

$$
\left\langle f\left(\mathbf{r} ; \mathbf{r}_{1}, \ldots, \mathbf{r}_{N}\right)\right\rangle=\int \ldots \int d V_{1} \ldots d V_{N} p\left(\mathbf{r}_{1}, \ldots, \mathbf{r}_{N}\right) f\left(\mathbf{r} ; \mathbf{r}_{1}, \ldots, \mathbf{r}_{N}\right),
$$

and the partial configurational average with one inclusion held fixed is defined by

$$
\begin{aligned}
\left\langle f\left(\mathbf{r} \mid \mathbf{r}_{i} ; \mathbf{r}_{1}, \ldots, \ldots, \mathbf{r}_{N}\right)\right\rangle= & \int \ldots \int d V_{1} \ldots{ }^{\prime} \ldots d V_{N} p\left(\mathbf{r}_{1}, \ldots, \ldots, \mathbf{r}_{N} \mid \mathbf{r}_{i}\right) f \\
& \times\left(\mathbf{r} \mid \mathbf{r}_{i} ; \mathbf{r}_{1}, \ldots, \ldots, \mathbf{r}_{N}\right),
\end{aligned}
$$


where the first coordinate $\mathbf{r}$ indicates the field point of evaluation, and the $\left(\mathbf{r}_{1}, \ldots, \mathbf{r}_{N}\right)$ or $\left(\mathbf{r}_{1}, \ldots, \ldots, \mathbf{r}_{N}\right)$ indicates the dependence of the random function on the specific configuration chosen.

The total field at any point outside all inclusions can be given in the multiple scattering form

$$
\begin{aligned}
\mathbf{u}\left(r ; r_{1}, r_{2}, \ldots, r_{N}\right)= & \mathbf{u}^{\mathrm{i}}(r)+\sum_{k=1}^{N} \mathbf{T}^{\mathrm{s}}\left(r_{k}\right) \mathbf{u}^{\mathrm{i}}(r) \\
& +\sum_{m=1}^{N} \mathbf{T}^{\mathrm{s}}\left(r_{m}\right) \sum_{k=1, k \neq m}^{N} \mathbf{T}^{\mathrm{s}}\left(r_{k}\right) \mathbf{u}^{\mathrm{i}}(r)+\ldots,
\end{aligned}
$$

where the single summation denotes the primary scattered terms, the double summation the secondary terms and so on. As shown in Figure 2, the primary scattering is due to the incident waves alone, and the second scattering represents the rescattering of the primary scattered waves, etc. This expression accounts completely for the interaction among the distributed particles. However, the scattering field expressed in the multiple scattering form is difficulty to deal with in order to predict the effective properties. So we introduce the effective exciting field on $j$-th inclusion

$$
\begin{aligned}
\mathbf{u}^{\mathrm{e}}\left(r \mid r_{j} ; r_{1}, r_{2}, \ldots, r_{N}\right)= & \mathbf{u}^{\mathrm{i}}(r)+\sum_{k \neq j}^{N} T\left(r_{k}\right) \mathbf{u}^{\mathrm{i}}(r) \\
& +\sum_{m \neq j}^{N} T\left(r_{m}\right) \sum_{k \neq m}^{N} T\left(r_{k}\right) \mathbf{u}^{\mathrm{i}}(r)+\ldots,
\end{aligned}
$$

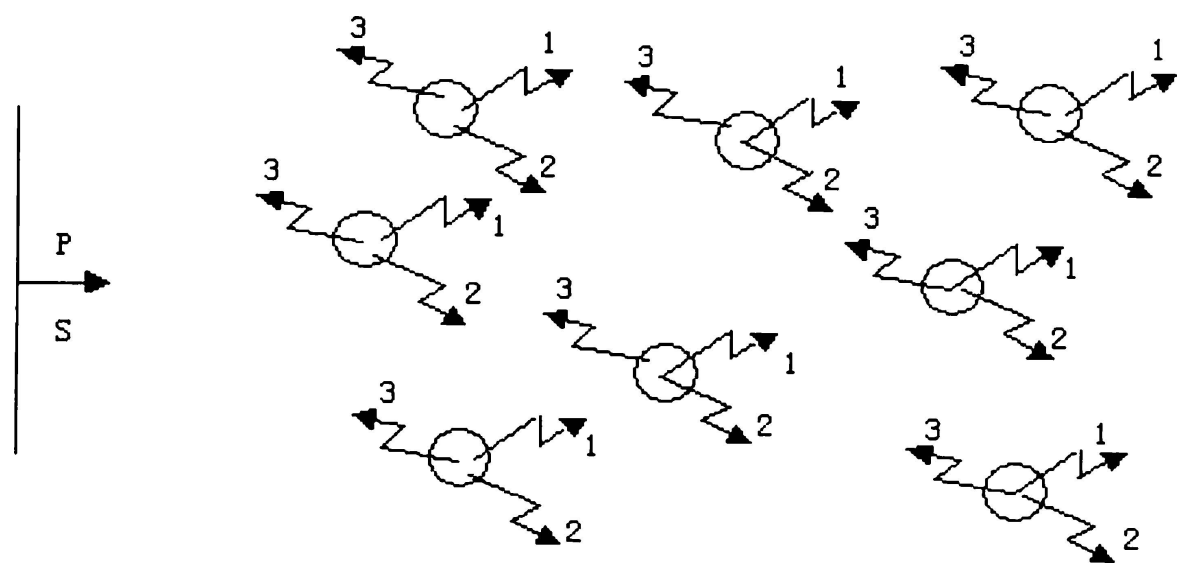

Figure 2. The multiple-scattered waves by the distributed inclusions. 
which is the counterpart of total field with $j$-th inclusion absent. Then, we can rewrite the total field as

$$
\mathbf{u}\left(\mathbf{r} ; \mathbf{r}_{1}, \ldots, \mathbf{r}_{N}\right)=\mathbf{u}^{\mathrm{i}}(\mathbf{r})+\sum_{k=1}^{N} \mathbf{T}\left(r_{k}\right) \mathbf{u}^{\mathrm{e}}\left(\mathbf{r} \mid \mathbf{r}_{k} ; \mathbf{r}_{1}, \ldots, \ldots, \mathbf{r}_{N}\right) .
$$

Similarly, we can write

$$
\mathbf{u}^{\mathrm{e}}\left(\mathbf{r} \mid \mathbf{r}_{\mathrm{i}} ; \mathbf{r}_{1}, \ldots, \ldots, \mathbf{r}_{N}\right)=\mathbf{u}^{\mathrm{i}}(\mathbf{r})+\sum_{k \neq i}^{N} \mathbf{T}\left(r_{k}\right) \mathbf{u}^{\mathrm{e}}\left(\mathbf{r} \mid \mathbf{r}_{i}, \mathbf{r}_{k} ; \mathbf{r}_{1}, \ldots \ldots, \ldots, \mathbf{r}_{N}\right)
$$

where $\left(\mathbf{r}_{1}, \ldots{ }^{\prime} \ldots, \mathbf{r}_{N}\right)$ means the absent of two inclusions. In turn, the $\mathbf{u}^{\mathrm{e}}\left(\mathbf{r} \mid \mathbf{r}_{\mathbf{i}}, \mathbf{r}_{k} ; \mathbf{r}_{1}, \ldots{ }^{\prime} \ldots, \mathbf{r}_{N}\right)$ can be write in term of the effective exciting field with the absent of three inclusions, and so on. Then, a set of equations in hierarchy are formed. Truncating these equations to obtain an approximate solution usually resorts to the self-consistent approximation proposed by Foldy (1945)

$$
\left\langle\mathbf{u}\left(\mathbf{r} ; \mathbf{r}_{1}, \ldots, \mathbf{r}_{N}\right)\right\rangle=\left\langle\mathbf{u}^{\mathrm{e}}\left(\mathbf{r} \mid \mathbf{r}_{k} ; \mathbf{r}_{1}, \ldots \ldots, \mathbf{r}_{N}\right)\right\rangle
$$

and the quasi-crystalline approximation proposed by Lax (1951)

$$
\left\langle\mathbf{u}^{\mathrm{e}}\left(\mathbf{r} \mid \mathbf{r}_{k} ; \mathbf{r}_{1}, \ldots{ }^{\prime} \ldots, \mathbf{r}_{N}\right)\right\rangle=\left\langle\mathbf{u}^{\mathrm{e}}\left(\mathbf{r} \mid \mathbf{r}_{i}, \mathbf{r}_{k} ; \mathbf{r}_{1}, \ldots{ }^{\prime} \ldots, \mathbf{r}_{N}\right)\right\rangle .
$$

After the statistical average is performed on Equation (25) and the self-consistent approximation is used, Foldy obtained the effective wavenumber of the coherent wave

$$
\left(\frac{k_{*}}{k_{0}}\right)^{2}=1+\frac{4 \pi n}{k_{0}^{2}} F\left(k_{0}\right)
$$

where $F\left(k_{0}\right)$ is the frequency-dependent isotropic scattering amplitude of an individual scatterer. $k_{0}$ is the wavenumber of the incident wave and $k_{*}$ is the effective wavenumber of the coherent plane wave. It is noticed that only the statistic distribution information, namely, the number density $n\left(\mathbf{r}_{i}\right)=N p\left(\mathbf{r}_{i}\right)$, is used in the Foldy's equation. However, if the statistical average is performed on Equation (26) and the quasi-crystalline approximation is used, the partial number density $n\left(\mathbf{r}_{j} \mid \mathbf{r}_{i}\right)=(N-1) p\left(\mathbf{r}_{j} \mid \mathbf{r}_{i}\right)$ will be involved. Due to the complicated paircorrelation function involved, no explicit expression of the effective wavenumber can be obtained in general. In view of this disadvantage and considering that the higher order statistic information is usually unavailable apart from the volume 
concentration, Lax modified the Foldy's equation by introducing a correction factor

$$
\left(\frac{k_{*}}{k_{*}}\right)^{2}=1+\frac{4 \pi n}{k_{0}^{2}} c^{\prime} F\left(k_{0}\right)
$$

Either Foldy's equation (29) or Lax's equation (30) consider only the forward scattering amplitude of an individual inclusion. Waterman (1961) further considered the contribution of the backward scattering amplitude and gave an alternative formula based on the double plane waves theory

$$
\left(\frac{k_{*}}{k_{0}}\right)^{2}=\left[1+\frac{2 \pi n}{k_{0}^{2}} F\left(k_{0}, 0\right)\right]^{2}-\left[\frac{2 \pi n}{k_{0}^{2}} F\left(k_{0}, \pi\right)\right]^{2},
$$

where $F\left(k_{*}, 0\right)$ and $F\left(k_{*}, \pi\right)$ are the forward and backward scattering amplitudes of an individual inclusion. It is noticed that Waterman's equation reduces to Foldy's equation for isotropic scattering, namely, $F\left(k_{*}, 0\right)=F\left(k_{*}, \pi\right)$. For the elastic waves propagating through an inhomogeneous medium, Gubernatis (1984) obtained the effective wavenumbers of the coherent plane waves based on the independent scattering approximation. Although the independent scattering approximation is rather different from the Foldy's approximation, the resulting equation evaluating the effective wavenumber is also only related to the number density of inclusions and the forward scattering amplitudes of an individual scatterer

$$
\begin{aligned}
& \left(\frac{k_{p *}}{k_{\mathrm{p} 0}}\right)^{2}=1+\left.\frac{4 \pi n}{k_{p 0}^{2}}\left[\mathbf{F}_{\mathrm{p}}\left(k_{0}, \theta, \phi\right) \cdot \mathbf{a}\right]\right|_{\theta=0}, \\
& \left(\frac{k_{\mathrm{s} *}}{k_{\mathrm{s} 0}}\right)^{2}=1+\left.\frac{4 \pi n}{k_{\mathrm{s} 0}^{2}}\left[\mathbf{F}_{\mathrm{s}}\left(k_{0}, \theta, \phi\right) \cdot \mathbf{b}\right]\right|_{\theta=0},
\end{aligned}
$$

where $k_{\mathrm{p} 0}$ and $k_{\mathrm{s} 0}$ are the wavenumbers of the incident longitudinal and shear waves. $k_{\mathrm{p} *}$ and $k_{\mathrm{s} *}$ are the effective wavenumbers of the coherent plane longitudinal and shear waves. $\mathbf{F}_{\mathrm{p}}\left(k_{0}, \theta, \phi\right)$ and $\mathbf{F}_{\mathrm{s}}\left(k_{0}, \theta, \phi\right)$, are the frequency- and azimuthdependent far-field scattering amplitude. In the present case they can be expressed as

$$
\begin{aligned}
{\left.\left[\mathbf{F}_{\mathrm{p}}\left(k_{0}, \theta, \phi\right) \cdot \mathbf{a}\right]\right|_{\theta=0}=} & \sum_{n=0}^{\infty}(-\mathrm{i})^{n} A_{0 n}^{\mathrm{s}}, \\
{\left.\left[\mathbf{F}_{\mathrm{s}}\left(k_{0}, \theta, \phi\right) \cdot \mathbf{b}\right]\right|_{\theta=0}=} & \sum_{n=1}^{\infty} \frac{(-\mathrm{i})^{n}}{2}\left\{n(n+1) C_{1 n}^{\mathrm{s}}-C_{-1 n}^{\mathrm{s}}\right. \\
& \left.+\frac{n(n+1)}{k_{\mathrm{s}}^{0}} B_{1 n}^{\mathrm{s}}+\frac{1}{k_{\mathrm{s}}^{0}} B_{-1 n}^{\mathrm{s}}\right\} .
\end{aligned}
$$


Because the far-field scattering amplitudes are complex-valued and frequencydependent, $k_{\mathrm{p} *}$ and $k_{\mathrm{s} *}$, are thus complex-valued and frequency-dependent. The real part of complex-valued wavenumber is related to the phase velocities and the imaginary part related to the attenuation.

$$
\begin{aligned}
& k_{\mathrm{p} *}(\omega)=k_{\mathrm{p} *}^{\mathrm{r}}(\omega)+\mathrm{i} k_{\mathrm{p} *}^{\mathrm{i}}(\omega)=\omega / c_{\mathrm{p}}^{*}+\mathrm{i} \alpha_{\mathrm{p}}^{*}, \\
& k_{\mathrm{s} *}(\omega)=k_{\mathrm{s} *}^{\mathrm{r}}(\omega)+\mathrm{i} k_{\mathrm{s} *}^{\mathrm{i}}(\omega)=\omega / c_{\mathrm{s}}^{*}+\mathrm{i} \alpha_{\mathrm{s}}^{*},
\end{aligned}
$$

where $c_{\mathrm{p}}^{*}, c_{\mathrm{s}}^{*}, \alpha_{\mathrm{p}}^{*}$ and $\alpha_{\mathrm{s}}^{*}$ are the phase velocities and the attenuations of the coherent $\mathrm{P}$ and $\mathrm{S}$ waves, respectively. Further, the real and imaginary parts of the complexvalued wavenumber are not independent but connected by the non-local KramerKroving relations (Donnell, 1981)

$$
\begin{aligned}
& k_{*}^{\mathrm{r}}(\omega)=\frac{2}{\pi} p \cdot v \cdot \int_{0}^{\infty} \frac{\omega^{\prime} k_{*}^{\mathrm{i}}(\omega)}{\omega^{\prime 2}-\omega^{2}} d \omega^{\prime}, \\
& k_{*}^{\mathrm{i}}(\omega)=-\frac{2}{\pi} p \cdot v \cdot \int_{0}^{\infty} \frac{\omega k_{*}^{\mathrm{r}}\left(\omega^{\prime}\right)}{\omega^{\prime 2}-\omega^{2}} d \omega^{\prime},
\end{aligned}
$$

where $p . v$. denotes principal value integral. The complex-valued propagation constant means an attenuated wave. In other word, the coherent plane waves propagating through the particle-reinforced composites will be attenuated due to the multiple scattering effects among the inclusions. We know that the general plane waves propagaating through a dissipative medium are of the complex-valued wavenumbers. However, the complex-valued wavenumbers result from the energy absorption in the dissipative medium but the energy diffusion in the elastic inhomogeneous medium. The complex-valued and frequency-dependent dynamic effective elastic moduli of the composites can be obtained from the effective wavenumbers by

$$
\begin{aligned}
& \mu_{*}(\omega)=\mu_{*}^{\mathrm{r}}(\omega)+\mathrm{i} \mu_{*}^{\mathrm{i}}(\omega)=\mu_{0} \frac{\rho_{*}}{\rho_{0}}\left(\frac{k_{\mathrm{s} 0}}{k_{\mathrm{s} *}}\right)^{2}, \\
& \lambda_{*}(\omega)=\lambda_{*}^{\mathrm{r}}(\omega)+\mathrm{i} \lambda_{*}^{\mathrm{i}}(\omega)=\left(\lambda_{0}+2 \mu_{0}\right) \frac{\rho_{*}}{\rho_{0}}\left(\frac{k_{\mathrm{p} 0}}{k_{\mathrm{p} *}}\right)^{2}-2 \mu_{0} \frac{\rho_{*}}{\rho_{0}}\left(\frac{k_{\mathrm{s} 0}}{k_{\mathrm{s} *}}\right)^{2},
\end{aligned}
$$

where the effective mass density of the composites, $\rho_{*}$, can be obtained directly from the volume average, namely,

$$
\rho_{*}=c \rho_{1}+(1-c) \rho_{0}
$$

where $c$ is the volume concentration of inclusions. 
Table I. Constituent properties of composites: Lead-Epoxy and Glass-Epoxy

\begin{tabular}{lccccc}
\hline Materials & $\lambda_{0}(\mathrm{GPa})$ & $\mu_{0}(\mathrm{GPa})$ & $\rho\left(\mathrm{kg} / \mathrm{m}^{3}\right)$ & $c_{\mathrm{p}}(\mathrm{m} / \mathrm{s})$ & $c_{\mathrm{s}}(\mathrm{m} / \mathrm{s})$ \\
\hline Epoxy & 4.59 & 1.57 & $1.18 \mathrm{E} 3$ & 2559 & 1153 \\
Lead & 38.76 & 8.35 & $11.3 \mathrm{E} 3$ & 2215 & 860 \\
Glass & 17.11 & 29.9 & $2.55 \mathrm{E} 3$ & 5492 & 3424 \\
\hline
\end{tabular}

From Datta et. al. (1988).

\section{Numerical Results and Discussion}

In the present numerical study, we use Equation (32) obtained by Gubernatis based on the independent scattering approach. The dynamic effective properties, i.e. the effective phase velocities, the effective attenuation and the effective elastic moduli, are predicted for two kinds of composite materials, namely, Lead-Epoxy and GlassEpoxy. The constituent properties of these composites are listed in Table I. In order to examine the effects of viscoelastic matrix on the dynamic effective properties, the standard solid model of a viscoelastic material is used. The relaxation functions and their corresponding complex moduli are

$$
\begin{aligned}
\mu_{2}(t) & =\mu_{\infty}+\left(\mu_{0}-\mu_{\infty}\right) \exp \left(-t / \tau_{\mu}\right) ; \\
\hat{\mu}_{2}(\omega)=\mathrm{i} \omega F\left(\mu_{2}(t)\right) & =\left(\mu_{\infty}-\mathrm{i} \mu_{0} \omega \tau_{\mu}\right) /\left(1-\mathrm{i} \omega \tau_{\mu}\right), \\
\lambda_{2}(t) & =\lambda_{\infty}+\left(\lambda_{0}-\lambda_{\infty}\right) \exp \left(-t / \tau_{\lambda}\right) ; \\
\hat{\lambda}_{2}(\omega)=\mathrm{i} \omega F\left(\lambda_{2}(t)\right) & =\left(\lambda_{\infty}-\mathrm{i} \lambda_{0} \omega \tau_{\lambda}\right) /\left(1-\mathrm{i} \omega \tau_{\lambda}\right),
\end{aligned}
$$

where $\mu_{0}$ and $\lambda_{0}$ are the shear and the dilatational short-term moduli, respectively. $\mu_{\infty}$ and $\lambda_{\infty}$ are the shear and the dilatational long-term moduli, respectively. In this numerical example, it is assumed $\mu_{0} / \mu_{\infty}=\lambda_{0} / \lambda_{\infty}=2$. $\tau_{\lambda}$ and $\tau_{\mu}$ are the relaxation times and are assumed $\tau_{\lambda}=\tau_{\mu}=0.001 \mathrm{~s}$. It is noticed that the elastic material can be recovered by letting $\tau_{\lambda}=\tau_{\mu}=\infty$. For purpose of comparing the material dissipation effects with the multiple scattering effects, three cases are considered: 1) The elastic matrix reinforced by distributed inclusions with the volume concentration $c=0.15$.2) The viscoelastic matrix reinforced by distributed particles with the volume concentration $c=0.15$.3) The homogeneous viscoelastic matrix without any inclusion.

In Figure 3, the predicted effective wavenumbers $\left(k_{\mathrm{p} *}, k_{\mathrm{s} *}\right)$ as a function of nondimensional wavenumber $\left(k_{\mathrm{s} 0} a\right)$ are shown for Lead-Epoxy composites with the volume concentrations $c=0.05$ and 0.15 . Here, the viscoelasticity of matrix is not taken into accounted. The numerical results obtained from the present work are the same with that obtained by Datta et al. (1988). Hence, the validity of the FORTRAN codes in the present work for evaluating the effective wavenumbers is verified.

The frequency-dependent complex-valued moduli of the viscoelastic matrix are shown in Figure 4. It is shown that the imaginary part of the complex moduli, which 


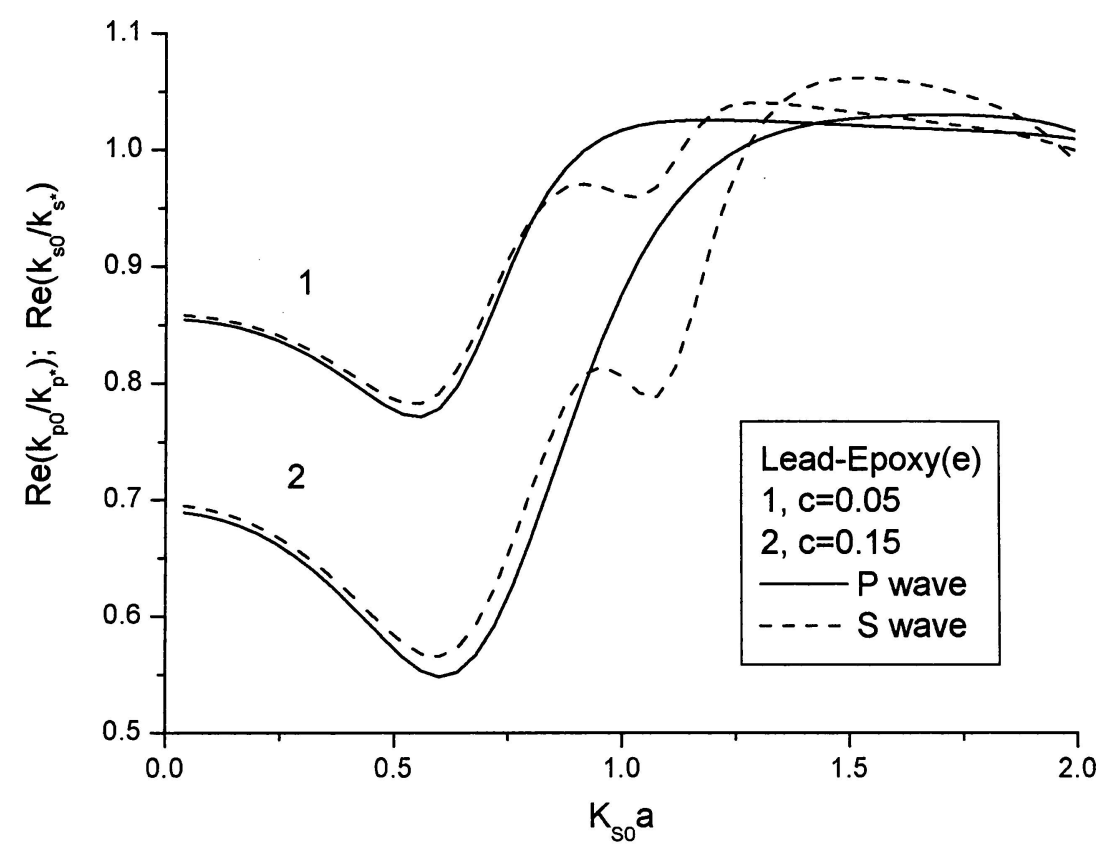

(a)

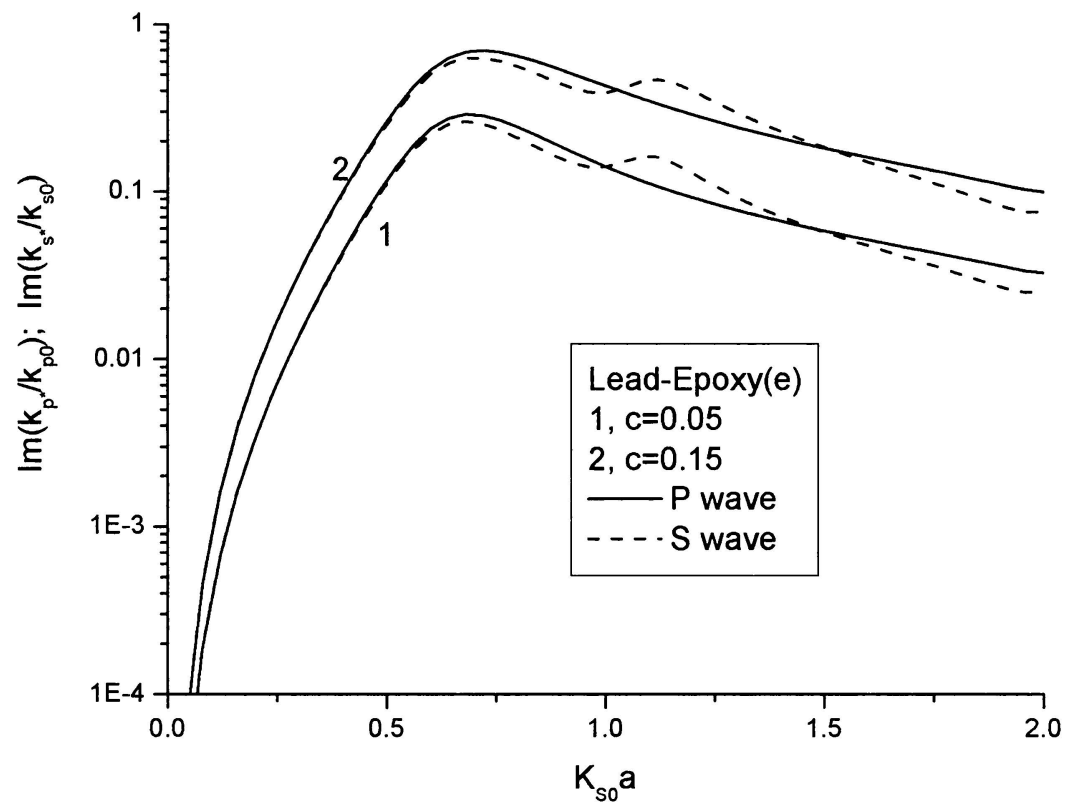

(b)

Figure 3. The normalized phase velocities and attenuation in Lead-Epoxy composite with elastic matrix. 


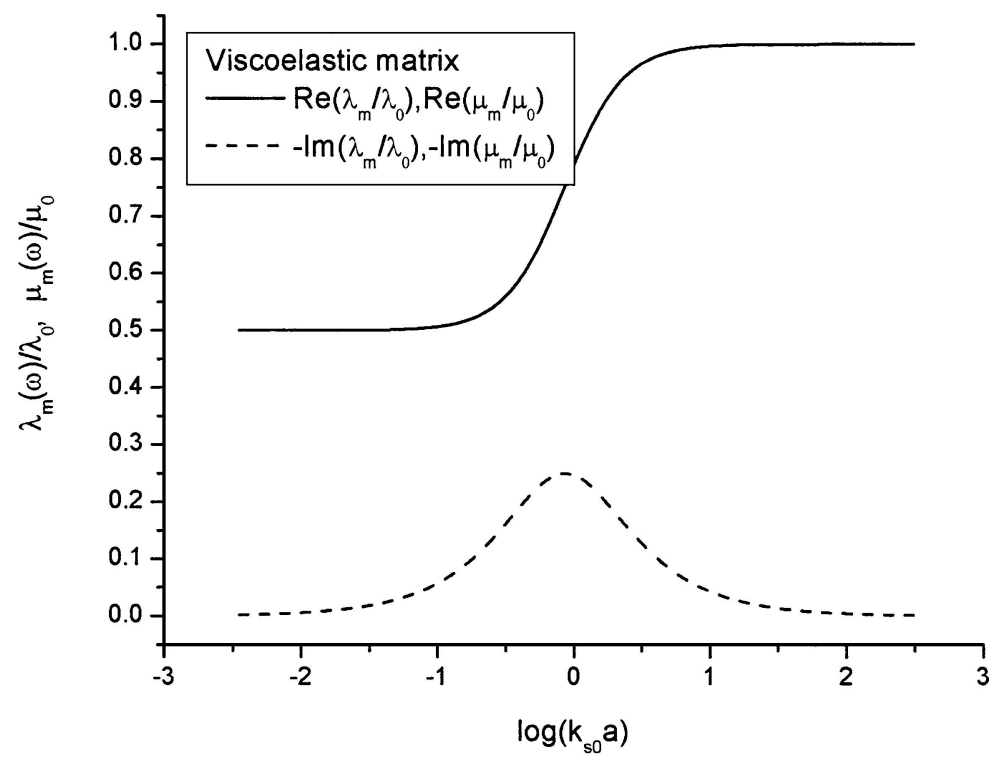

Figure 4. The frequency-dependent complex moduli of the viscoelastic matrix $\left(\mu_{0} / \mu_{\infty}=\right.$ $\left.\lambda_{0} / \lambda_{\infty}=2 ; \tau=0.001 \mathrm{~s} ; \lambda_{m}(\omega)=\hat{\lambda}_{2}(\omega) ; \mu_{m}(\omega)=\hat{\mu}_{2}(\omega)\right)$.

indicates the viscosity of material, is restricted to a finite frequency range if the standard linear solid model is used. At a relatively low and high frequencies the viscoelastic matrix reduces to the elastic matrix with the long and the short-term moduli, respectively.

In Figures 5(a) and 5(b), the predicted effective phase velocities and attenuation are shown for Lead-Epoxy composites with the volume concentration $c=0.15$. It is noticed that the phase velocities of $\mathrm{P}$ and $\mathrm{S}$ waves decrease, but the attenuations of $\mathrm{P}$ and $\mathrm{S}$ waves increase at a relatively low frequency due to the viscosity of matrix. However, the viscous effects of matrix decrease gradually with the increase of frequency. The predicted effective elastic moduli are shown in Figures 6(a) and 6(b). It can be seen that the viscosity of matrix can affect the effective elastic moduli significantly at a relatively low frequency but the influence decreases gradually when the frequency increases. Moreover, the dissipative effects of the viscoelastic matrix and the multiple scattering effect of the distributed inclusions are both contributed to the attenuation of waves at a relatively low frequency. But their effects decreases gradually when frequency increase.

In Figures 7(a) and 7(b), the predicted effective phase velocities and attenuation are shown for Glass-Epoxy composites with the volume concentrations $c=0.15$. Compared with the heavy particle (lead) case, the light particle (glass) case shows evident different features. The effective phase velocities of the composites with the distributed heavy particles (lead) decrease relative to the phase velocities of 


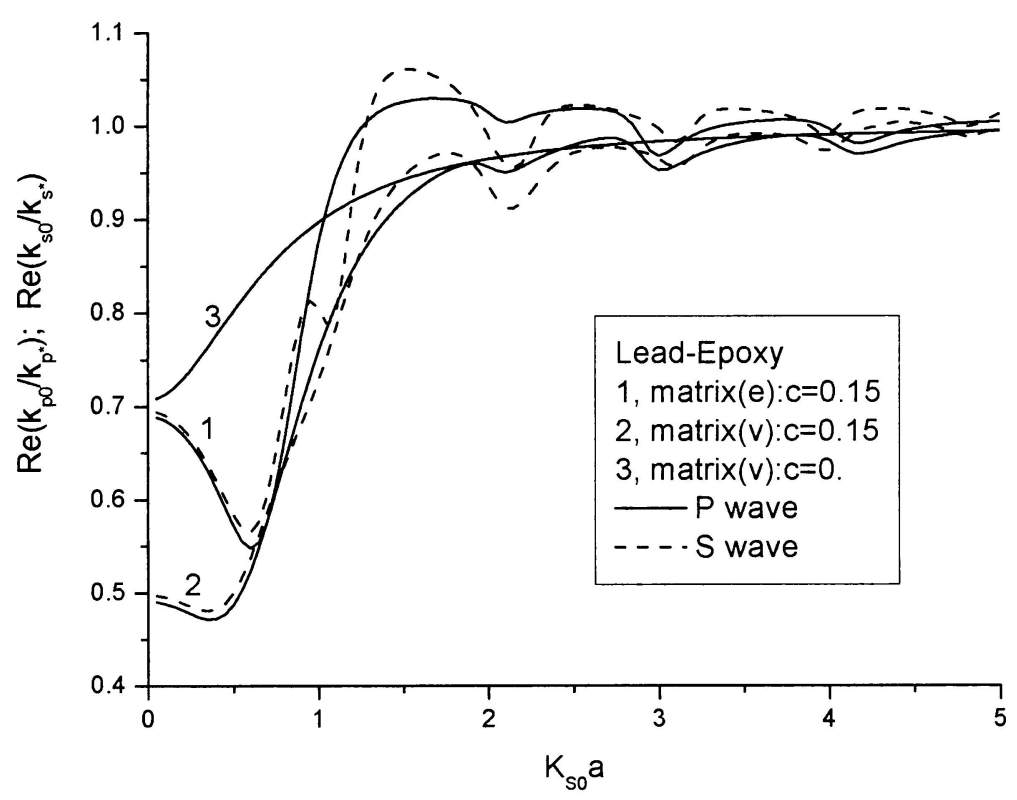

(a)

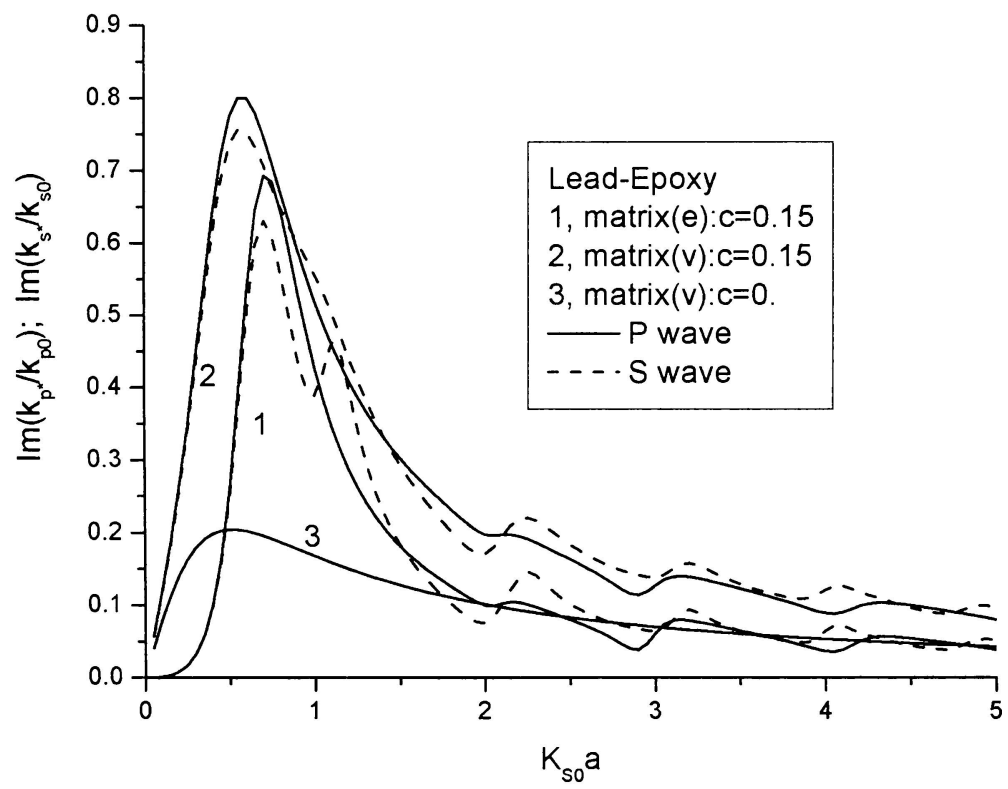

(b)

Figure 5. The normalized phase velocities and attenuation of Lead-Epoxy composites with the elastic and viscoelastic matrix. 


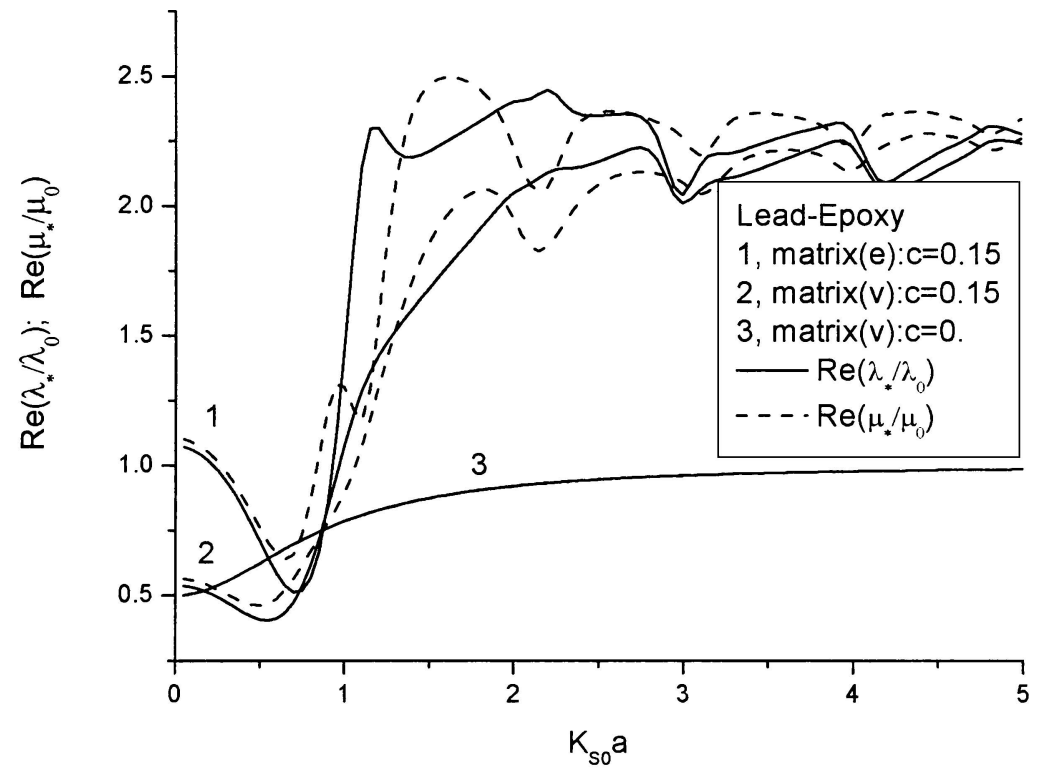

(a)

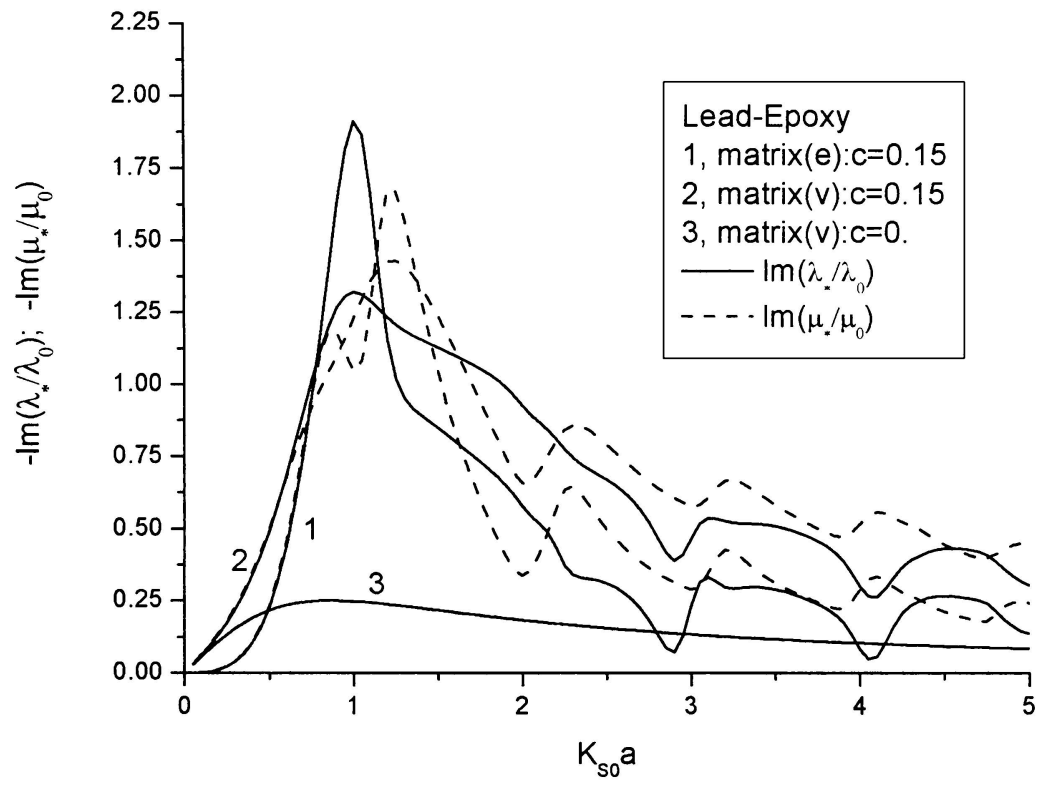

(b)

Figure 6. The normalized frequency-dependent effective elastic moduli of Lead-Epoxy composites with the elastic and viscoelastic matrix. 

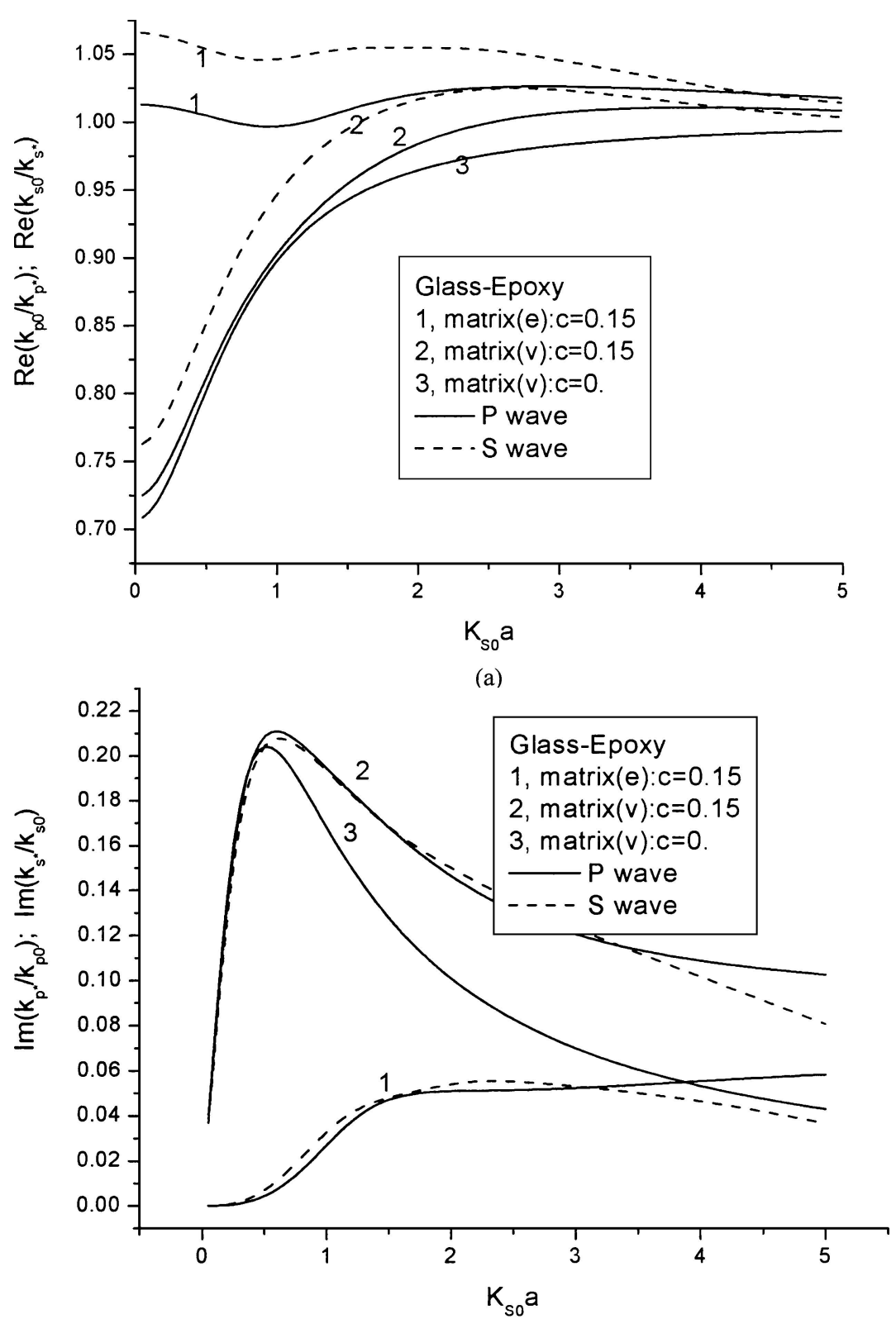

(b)

Figure 7. The normalized phase velocities and attenuation of Glass-Epoxy composite with the elastic and viscoelastic matrix. 


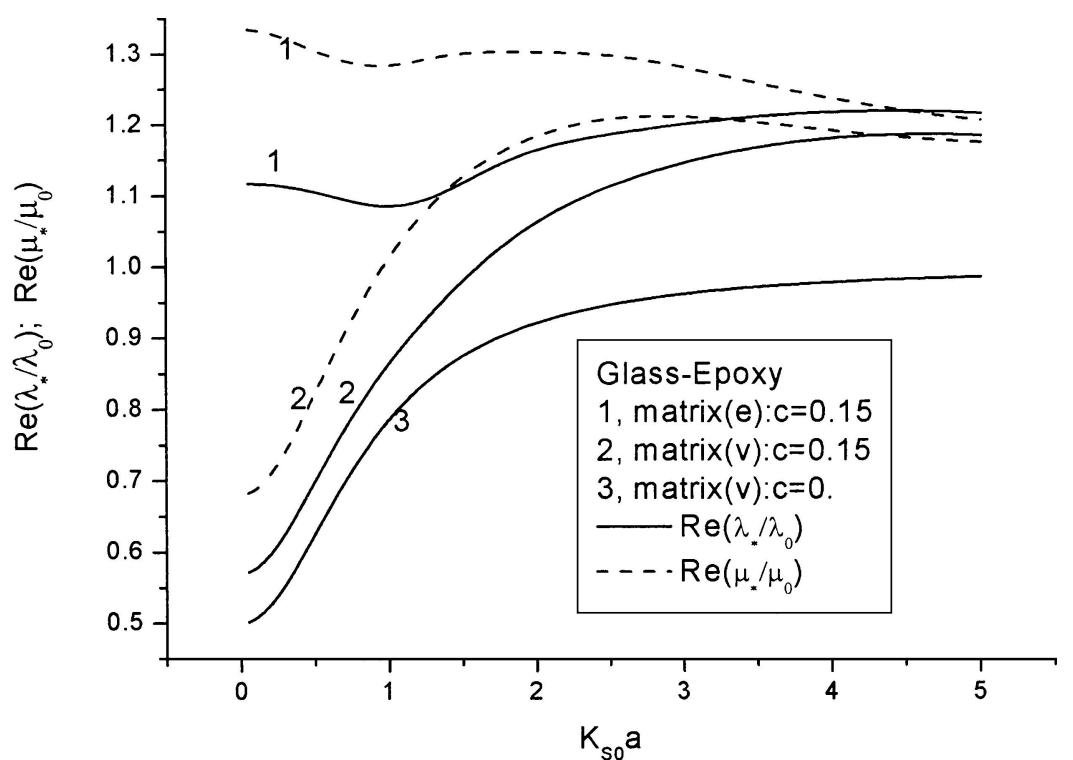

(a)

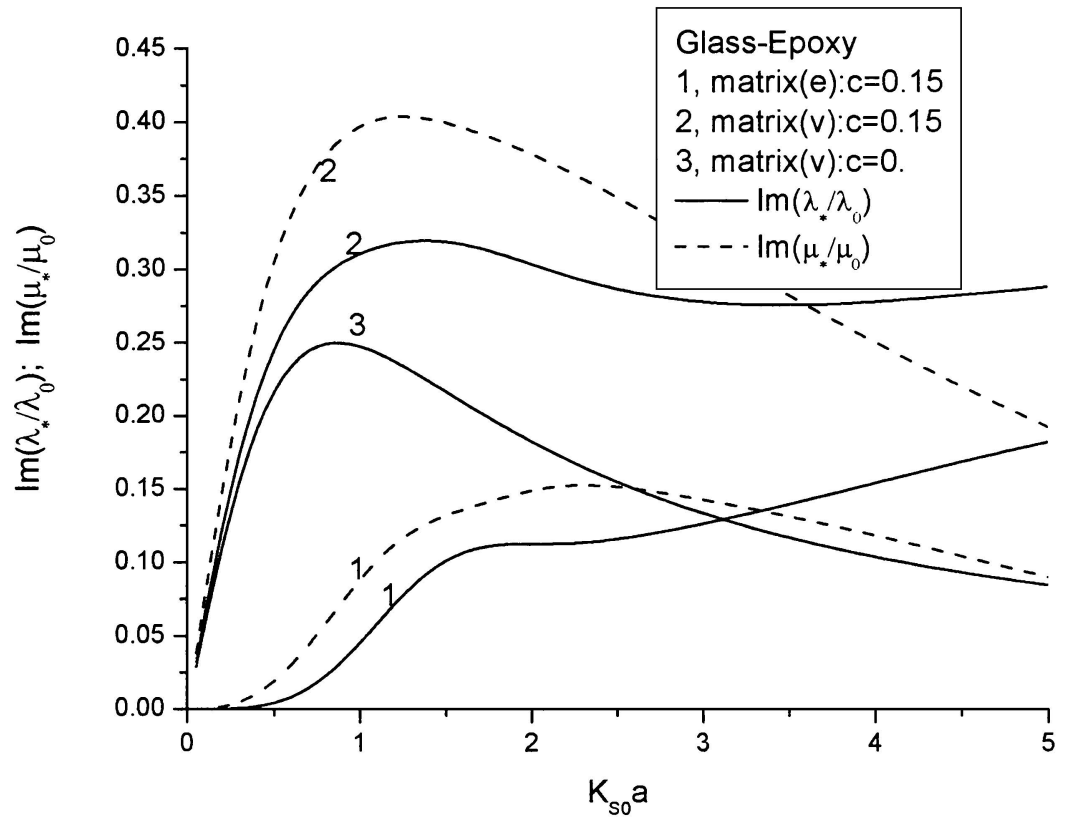

(b)

Figure 8. The normalized frequency-dependent effective moduli of Glass-Epoxy composites with the elastic and viscoelastic matrix. 
homogeneous matrix at a relatively low frequency. However, the effective phase velocities of the composites with the distributed light particles (glass) increase relative to the phase velocities of homogeneous matrix. Moreover, the effects of multiple scattering on the attenuation of waves are pronounced at a relatively low frequency for the composites with heavy particles but at a relatively high frequency for the composites with light particles. In both cases of the heavy particle and the light particles, the effects of material dissipation on the attenuation of waves are pronounced at a relatively low frequency and decrease gradually as the frequency increases. The enhanced effective elastic moduli of the composites with heavy particles are more pronounced than the composites with light particles at a relative high frequency, as shown in Figures 6 and 8.

\section{Concluding Remarks}

Even though the matrix and the reinforced particles are both elastic, the effective wavenumbers and the effective elastic moduli of composites are complex-valued and frequency-dependent. This feature can be attributed to the multiple scattering effects among the distributed particles. When the viscoelasticity of matrix, which is applicable for most of polymer matrix, is taken into account, the frequencydependent complex-valued effective wavenumbers and the effective elastic moduli are related to not only the multiple scattering among distributed particles but also the intrinsic dissipation of the viscoelastic material. However, their effects on the dynamic effective properties are different. The numerical predictions given for Lead-Epoxy and Glass-Epoxy composites show that the effects of viscous dissipation are most pronounced at a relatively low frequency and decrease gradually as the frequency increases. The effects of multiple scattering on the attenuation are pronounced at a relatively high frequency for the composites reinforced by the light particle but at a relatively low frequency for the composites reinforced by the heavy particle. The difference can be accounted for by the low-frequency resonance of the heavy particle. The low-frequency resonance of the heavy particle can also account for the drastic drop in the effective phase velocities and the effective elastic moduli of composites at a relatively low frequency.

\section{Acknowledgement}

This work was supported by National Natural Science Foundation of China (No. 10272003) and Talent Foundation of University of Science \& Technology Beijing.

\section{References}

Berryman, J.G., 'Long-wavelength propagation in composite elastic media I spherical inclusions', J. Acoust. Soc. Am. 68, 1980, 1809-1819. 
Datta, S.K., Ledbetter, H.M. and Shindo, Y., 'Phase velocity and attenuation of plane elastic waves in a particle-reinforced composite medium', Wave Motion 10, 1988, 171-182.

Donnell, M.O., Jaynes, E.T. and Miller, J.G. 'Kramers-Kronig relationship between ultrasonic attenuation and phase velocity', J. Acoust. Soc. Am. 69, 1981, 696-701.

Foldy, L.O., 'The multiple scattering of waves', Phys. Rev. 67, 1945, 107-119.

Gubernatis, J.E., 'Effects of microstructure on speed and attenuation of elastic waves in porous materials', Wave Motion 6, 1984, 579-589.

Kanaun, S.K., 'Self-consistent methods in the propagation through heterogeneous media', in Heterogeneous Media - Micromechanics Modeling Methods and Simulations, K.Z. Markov and L. Preziosi (eds.), Birkhauser, Boston.

Lax, M., 'Multiple scattering of waves', Rev. Modern Phys. 23, 1951, 287-310.

Sabia, F.J. and Willis, J.R., 'A simple self-consistent analysis of wave propagation in particulate composites', Wave Motion 10, 1988, 127-142.

Shindo, Y., Nozaki, H. and Datta, S.K., 'Effect of interface layers on elastic wave propagation in a metal matrix composite reinforced by particles', ASME, J. Appl. Mech. 62, 1995, 178-185.

Varadan, V.K., Ma, Y. and Varadan, V.V., 'A multiple scattering theory for elastic waves propagation in discrete random medium', J. Acoust. Soc. Am. 77, 1985, 375-385.

Waterman, P.C. and Truell, R., 'Multiple scattering of waves', J. Math. Phys. 2, 1961, 512-537. 